\title{
Primary neuroendocrine tumors of the thymus: Clinical review of 22 cases
}

\author{
ZHENGBO SONG $^{1,2}$ and YIPING ZHANG ${ }^{1,2}$ \\ ${ }^{1}$ Department of Chemotherapy, Zhejiang Cancer Hospital; \\ ${ }^{2}$ Key Laboratory Diagnosis and Treatment Technology on Thoracic Oncology, Hangzhou, Zhejiang 310022, P.R. China
}

Received December 20, 2013; Accepted July 18, 2014

DOI: $10.3892 / \mathrm{ol} .2014 .2490$

\begin{abstract}
Primary neuroendocrine tumors of the thymus are rare mediastinum tumors, which present a distinct type of tumor, which exhibit morpholgical and biological neuroendorcine features including the production of numerous biogenic amines. The aim of the present study was to evaluate factors influencing long-term survival in patients with primary neuroendocrine tumors of the thymus. A total of 22 patients exhibiting primary thymic neuroendocrine tumors, who were treated at the Zhejiang Cancer Hospital (Hangzhou, China), between 1995 and 2012 were reviewed. Survival curves were plotted using the Kaplan-Meier method and the Cox proportional hazards model was used for multivariate analysis. The overall five-year survival rate was $45.5 \%$ and the median survival time was 59 months in all of the patients. Histological grade $(\mathrm{P}<0.001)$, Masaoka-Koga stage $(\mathrm{P}=0.003)$ and surgical resection status $(\mathrm{P}=0.004)$ were identified to be associated with patient survival time. Furthermore, multivariate analysis identified that the histological grade was an independent prognostic factor, which was applicable to all patients $(\mathrm{P}=0.009)$. Therefore, the histological grade and Masaoka-Koga stage, as well as surgical resection status present three prognostic factors in patients exhibiting primary thymic neuroendocrine tumors.
\end{abstract}

\section{Introduction}

Neuroendocrine tumors of the thymus are rare, with an annual incidence of $0.01 / 100,000$ in the USA (1). The histogenesis of neuroendocrine tumors varies and the tumor may arise from ectopic tissues in the mediastinum or present within the thymus (2). Thus, the histopathological classification, prognosis and treatment of primary neuroendocrine carcinomas of the thymus remain controversial.

Correspondence to: Dr Yiping Zhang, Department of Chemotherapy, Zhejiang Cancer Hospital, 38 Guangji Road, Hangzhou, Zhejiang 310022, P.R. China

E-mail: yipingzhang@yahoo.cn

Key words: thymus, neuroendocrine tumor, prognosis, treatment
According to the World Health Organization (WHO) (2), neuroendocrine tumors are included in the thymic carcinoma group and classified as two histopathological types; well-differentiated neuroendocrine carcinomas (typical and atypical carcinoid) and poorly differentiated neuroendocrine carcinomas (small cell carcinoma and large cell neuroendocrine carcinoma). The well-differentiated neuroendocrine carcinomas show a low grade of biological aggressiveness, while poorly differentiated neuroendocrine carcinomas are considered to be high-grade neuroendocrine tumors. As there have only been a small number of patients with neuroendocrine tumors of the thymus reported in the literature (3-7), a consensus has not been reached concerning the prognostic factors of primary neuroendocrine tumors of the thymus.

The aim of the present study was to evaluate the factors influencing long-term survival in 22 patients with primary neuroendocrine tumors of the thymus and to explore the role of various prognostic factors.

\section{Patients and methods}

Patient eligibility. The records of 22 patients exhibiting primary neuroendocrine tumors of the thymus, who were treated at the Zhejiang Cancer Hospital (Hangzhou, China), between 1995 and 2012, were reviewed. The 22 patients included 14 males and eight females, with a median age of 49.5 years. The histological type was determined according to the 2004 WHO classification (2) and the staging was performed for all patients according to the Masaoka-Koga system (8). Recurrence or metastases were identified using chest computed tomography (CT), as well as ultrasound and/or CT of the abdomen. The study was approved by the ethics committee of Zhejiang Cancer Hospital (Hangzhou, China).

Patient treatment. A total of ten patients underwent surgical resection following first diagnosis.A total of 9 patients received chemotherapy, 8 patients received radiation therapy, 3 patients received chemotherapy and radiotherapy and two patients received no futher treatment. The detailed treatment of the 22 patients is shown in Table I.

Follow-up. Patients were followed up every three to six months for the first five years, and once per year thereafter. Each patient's medical history, details of physical examinations and thoracic 


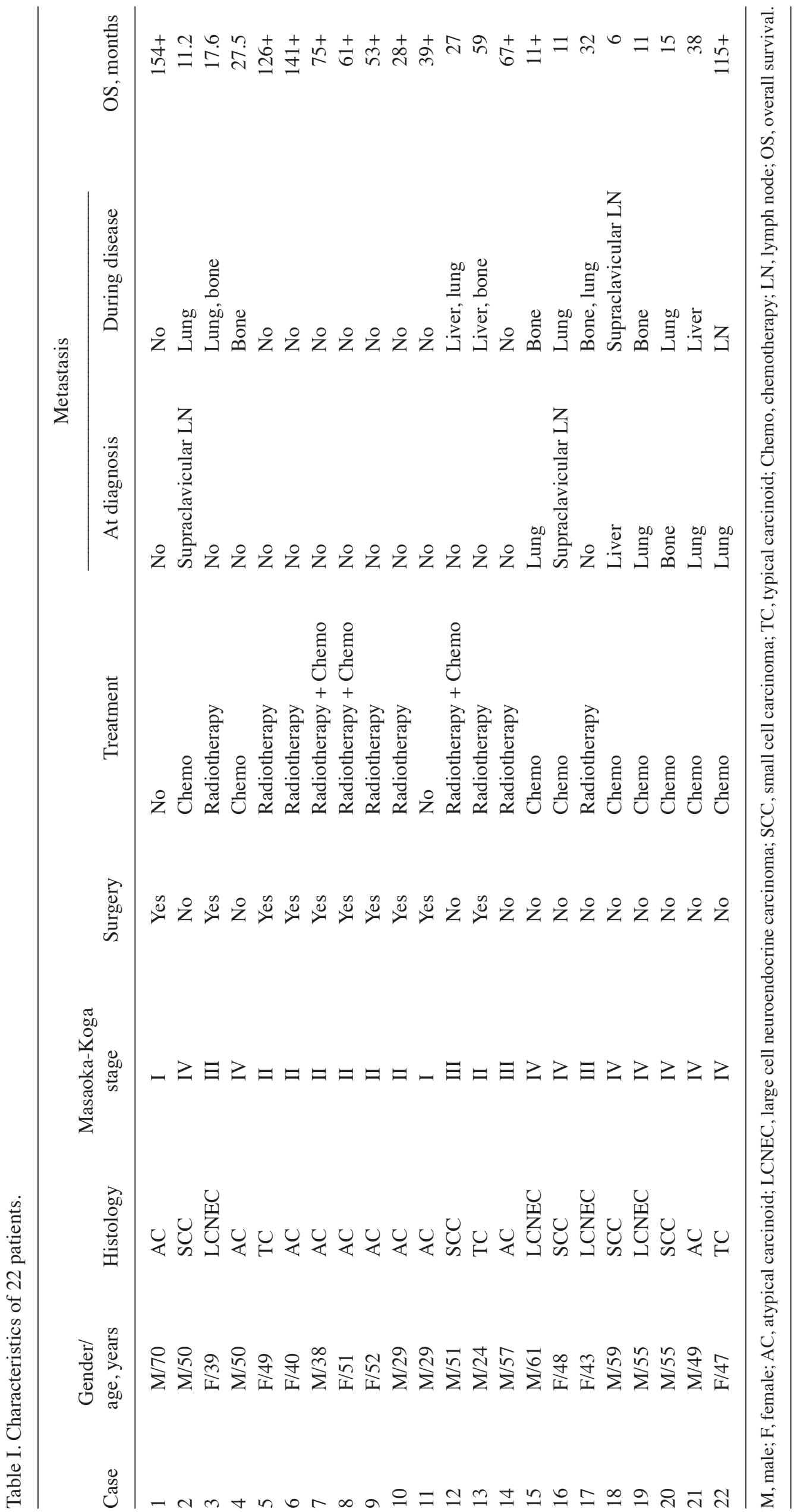


Table II. Univariate analysis of patient OS rate.

\begin{tabular}{lcc}
\hline Variable & Five-year OS rate, $\%$ & P-value \\
\hline Gender & & 0.311 \\
$\quad$ Male & 34.1 & \\
Female & 62.5 & 0.357 \\
Age, years & & \\
$\geq 50$ & 40.9 & \\
$\quad<50$ & 49.1 & 0.351 \\
Tumor size, cm & & \\
$>5$ & 40.1 & $<0.001$ \\
$\quad$ 55 & 50.0 & \\
Grade & & 0.003 \\
Poorly differentiated & 73.4 & \\
Well-differentiated & 0.00 & \\
Masaoka-Koga stage & & \\
I+II & 75.0 & \\
III+IV & 18.8 & \\
Surgery & & \\
Yes & 75.0 & \\
No & 18.8 & \\
\hline
\end{tabular}

OS, overall survival.

CT scans were recorded. The last follow-up was on Jan 30, 2013, with a median follow-up period for all patients of 109 months (range, 15-185 months).

Statistical analysis. Survival curves were calculated (using the Kaplan-Meier method) commencing from the date of the confirmed pathology to the date of mortality or the last follow-up. The log-rank test was used to compare overall survival (OS) time between different factors, including gender, age, tumor stage and surgery status. Multivariate analysis was performed using the Cox proportional hazards model and statistical analysis was performed using the SPSS version 15 software (SPSS, Inc., Chicago, IL, USA). Confidence intervals were calculated at the $95 \%$ level and $\mathrm{P}<0.05$ was considered to indicate a statistically significant difference.

\section{Results}

Clinical characteristics. The clinical characteristics of the 22 patients are listed in Table I. The 22 patients enrolled in the present study included 14 males and eight females, with a median age of 49.5 years. In total, 10 of the 22 individuals underwent surgery. The pathological stage was I and II in nine patients, and III and IV in 13 patients. According to the WHO criteria (2), based on the histopathological differentiation, all 22 cases were divided into two types; well-differentiated $(n=13)$ and poorly differentiated $(n=9)$ neuroendocrine carcinomas.

Survival analyses. Table II shows the results of the univariate analyses of the clinicopathological factors evaluated in the present study. At present, a total of 11 patients have survived,

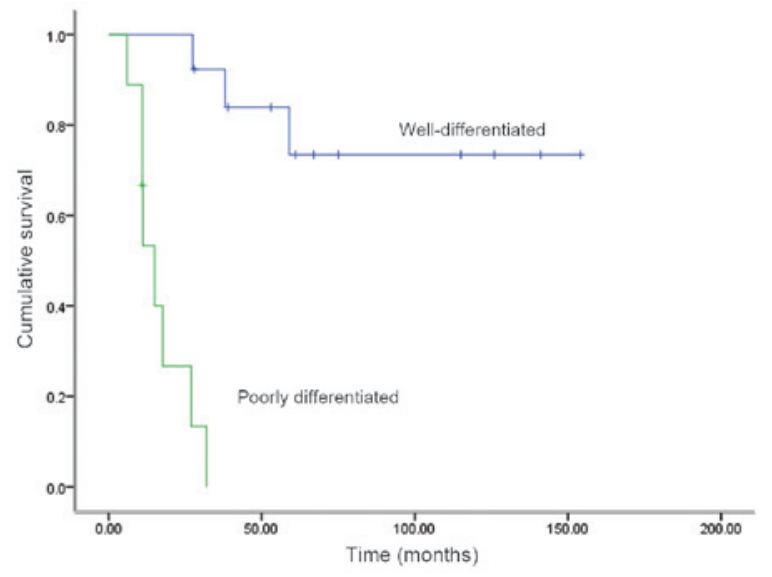

Figure 1. Kaplan-Meier curves comparing the survival times of patients with well- and poorly differentiated neuroendocrine carcinomas $(\mathrm{P}<0.001)$.

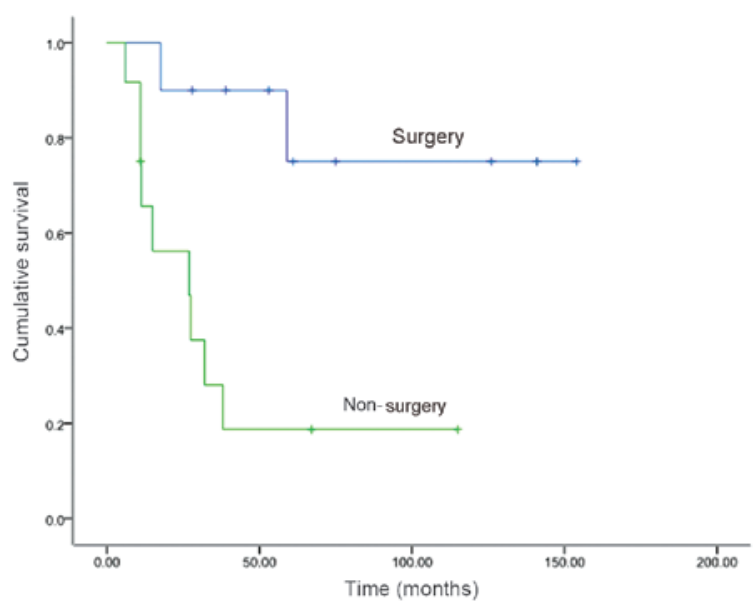

Figure 2. Kaplan-Meier curves comparing the survival time of patients that underwent surgery with those that did not $(\mathrm{P}=0.004)$.

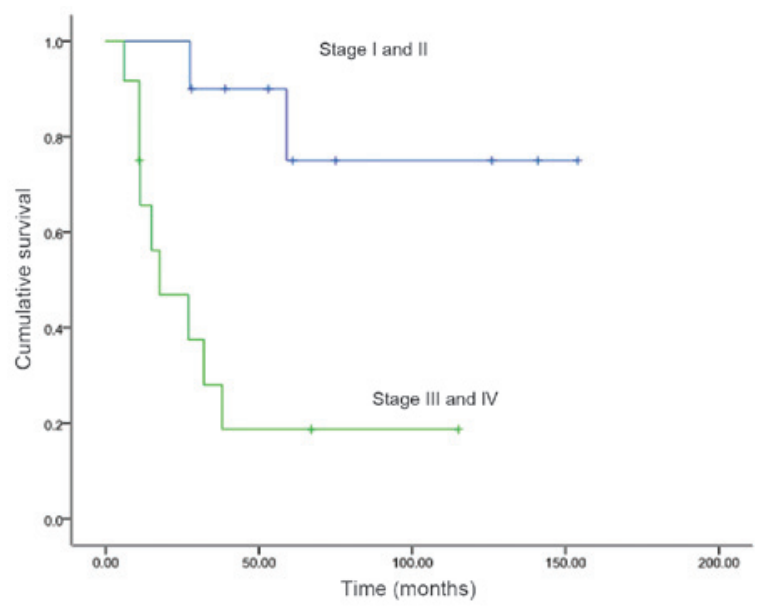

Figure 3. Kaplan-Meier curves comparing the survival time of patients with early (Stage I-II) and late (Stage III-IV) stage tumors $(\mathrm{P}=0.003)$.

however, 11 patients succumbed to the disease prior to the final follow up date. The median survival time for all patients was 59 months, and the five-year OS rate was $45.5 \%$, with ten patients surviving longer than five years. Patients with 
Table III. Multivariate analysis of patient overall survival rate.

\begin{tabular}{lccr}
\hline & \multicolumn{3}{c}{ Overall survival } \\
\cline { 2 - 4 } Variable & HR & 95\% CI & P-value \\
\hline Gender (male vs. female ) & 3.977 & $0.699-22.610$ & 0.120 \\
Age, years ( $\geq 50$ vs. $<50)$ & 0.968 & $0.880-1.065$ & 0.501 \\
Tumor size, cm $(>5$ vs. $\leq 5$ ) & 3.466 & $0.651-18.457$ & 0.145 \\
Grade (poorly differentiated vs. well-differentiated) & 51.074 & $2.698-966.717$ & 0.009 \\
Masaoka-Koga stage (III+IV vs. I+II) & 1.824 & $0.027-123.576$ & 0.780 \\
Surgery (yes vs. no) & 0.243 & $0.005-11.111$ & 0.469 \\
\hline
\end{tabular}

HR, hazards ratio; CI, confidence interval.

poorly differentiated neuroendocrine carcinomas, that were stage III and IV, and did not undergo surgery exhibited a significant correlation with poor OS (Table II and Figs. 1-3).

A multivariate Cox proportional hazards model was constructed, which accounted for age, gender, tumor size, histological grade and surgery as variable factors. The histological grade was the only independent prognostic factor identified for OS ( $\mathrm{P}=0.009$; Table III).

\section{Discussion}

Primary neuroendocrine tumors of the thymus are rare, with $\sim 400$ cases reported in the literature to date; the majority of which are case reports (3-7, 9-11). The median age at diagnosis has been relatively young in the majority of studies, ranging between 40 and 60 years. A male predominance has also been observed in the literature, which is consistent with the findings of the current report.

In a series of 15 patients reported by Fukai et al (5), the five-year survival rate was 33\%, and of the 14 cases reported by de Montpreville et al (3) the five-year survival rate was $31 \%$. The overall five-year survival rate in the present study was $45.5 \%$ (Table II), which is consistent with that reported in earlier studies $(3,5)$. However, the median OS was shorter than that of previous reports $(1,12,13)$, which may be due to more than half of the patients reported in the present study not undergoing surgery.

As the diagnosis of primary neuroendocrine tumors of the thymus is rare, only a small number of retrospective studies are available. Therefore, a standard therapeutic strategy has not yet been defined. Surgery remains the standard method for the treatment of thymic tumors compared with non-surgical options according to the Surveillance, Epidemiology, and End Results database analysis (1). In the present study, a significant difference in the OS of patients was identified between those who underwent surgery and those who did not. However, the prognostic factors currently in use for primary neuroendocrine tumors of the thymus, including histological grade, Masaoka-Koga grade and surgery status, remain controversial. To date, the histological grade, Masaoka-Koga stage and surgical resection status have been validated as prognostic factors. In addition, in the present study, carcinoids showed the optimum prognosis, while large cell neuroendocrine carcinoma and small cell carcinoma were associated with a poor prognosis (Fig. 1). Furthermore, patients with Masaoka-Koga stages III and IV showed a poorer prognosis than stage I and II patients (Table II and Fig. 3).

The major limitations of the current study were its retrospective nature and the subjects being obtained from a single institution. In addition, a small level of heterogeneity was identified among the surgical and non-surgical patients, which may have influenced the analysis of the prognosis. However, despite the small patient population that was used in this retrospective study, the results are considered to be meaningful.

In conclusion, thymic neuroendocrine tumors are associated with a discriminative prognosis. The histological grade, Masaoka-Koga stage and surgical resection status were identified to be prognostic factors. However, further study is required to fully validate the prognostic factors and determine a standard treatment for thymic neuroendocrine tumors.

\section{References}

1. Gaur P, Leary C and Yao JC: Thymic neuroendocrine tumors: a SEER database analysis of 160 patients. Ann Surg 251: 1117-1121, 2010

2. Travis WD, Brambilla E, Müller-Hermelink HK, et al (eds). WHO Classification of Tumours. Pathology \& Genetics of Tumours of the Lung, Pleura, Thymus and Heart. IARC Press, Lyon, pp145-147, 2004.

3. de Montpreville VT, Macchiarini P and Dulmet E: Thymic neuroendocrine carcinoma (carcinoid): a clinicopathologic study of fourteen cases. J Thorac Cardiovasc Surg 111: 134-141, 1996.

4. Cardillo G, Treggiari S, Paul MA, et al: Primary neuroendocrine tumours of the thymus: a clinicopathologic and prognostic study in 19 patients. Eur J Cardiothorac Surg 37: 814-818, 2010.

5. Fukai I, Masaoka A, Fujii Y, et al: Thymic neuroendocrine tumor (thymic carcinoid): a clinicopathologic study in 15 patients. Ann Thorac Surg 67: 208-211, 1999.

6. Moran CA and Suster S: Thymic neuroendocrine carcinomas with combined features ranging from well-differentiated (carcinoid) to small cell carcinoma. A clinicopathologic and immunohistochemical study of 11 cases. Am J Clin Pathol 113: 345-350, 2000.

7. Moran CA and Suster S: Neuroendocrine carcinomas (carcinoid tumor) of the thymus: a clinicopathologic analysis of 80 cases. Am J Clin Pathol 114: 100-110, 2000.

8. Koga K, Matsuno Y, Noguchi M, et al: A review of 79 thymomas: modification of staging system and reappraisal of conventional division into invasive and non-invasive thymoma. Pathol Int 44: 359-367, 1994.

9. Wang DY, Chang DB, Kuo SH, et al: Carcinoid tumours of the thymus. Thorax 49: 357-360, 1994. 
10. Brambilla E and Lantuejoul S: Thoracic neuroendocrine tumors. Ann Pathol 25: 529-544, 2005 (In French).

11. Gal AA, Kornstein MJ, Cohen C, et al: Neuroendocrine tumors of the thymus: a clinicopathological and prognostic study. Ann Thorac Surg 72: 1179-1182, 2001.

12. Moran CA: Primary neuroendocrine carcinomas of the mediastinum: review of current criteria for histopathologic diagnosis and classification. Semin Diagn Pathol 22: 223-229, 2005.
13. Cardillo G, Rea F, Lucchi M, et al: Primary neuroendocrine tumors of the thymus: a multicenter experience of 35 patients. Ann Thorac Surg 94: 241-246, 2012. 